\title{
Organic Light Emitting Pixel Arrays by Supra-molecular Self-organization
}

\author{
Ilenia Viola*, Marco Mazzeo, Alessandra Passabí, Stefania D’Amone, \\ Roberto Cingolani and Giuseppe Gigli*a \\ ${ }^{a}$ NNL, National Nanotechnology Laboratory of INFM, \\ c/o Dip. Ingegneria dell'Innovazione, Universitá di Lecce, via Arnesano, 73100 Lecce, Italy.
}

\begin{abstract}
We present a controlled, well-defined pattern replication of a micrometer template driven by a surface free energy lithographic technique, realized by molecular aggregation in dewetting conditions and by confining the liquid solution with geometric boundaries. The presented technique has allowed the fabrication of light-emitting devices, and in particular the realization of OLEDs with an array of addressable pixels.
\end{abstract}

Keywords: organic devices, self-assembling, confined geometry

\section{INTRODUCTION}

In the last years, several techniques for micro and submicron patterning of organic materials ${ }^{1,2}$ have been investigated for the fabrication of a novel class of optoelectronic devices, such as organic lasers ${ }^{3}$ and organic light emitting devices (OLEDs), ${ }^{4-6}$ exploiting light confinement effects as working prciples.

Besides, patterning molecular materials seems to be crucial in several application, such as integrated optical devices, full-color displays ${ }^{7}$ and lighting panels, ${ }^{8}$ where resolution improvement requires an increase of pixel integration.

Patterning could be also the solution for device degradation problems. The presence of defects is, in fact, considered the main reason of device damaging, because of their role in forming dendritic crystals or dark spots, spreading in the time through the deposited film for several microns. ${ }^{9}$

Several patterning approaches rely on conventional top-down lithographies. These are mainly based on the Nanoimprinting of organic layer, which consists in modifying a bulk material through a rigid stamp with fixed features. ${ }^{10,11}$

Besides, in the last years lithographic techniques exploiting the self-organization properties of molecular materials have been developed. These techniques, known as bottom-up approaches, are usually based on the dependence of the liquid behavior, in non-wetting conditions, on the surface free energy at the solid-liquid interface. In particular, a well-defined pattern can be realized by molecular aggregation in conditions of energy minimization and by confining the liquid solution by geometric boundaries. ${ }^{12,13}$

Bottom-up techniques appear extremely successful for the ease of realization, the well-defined control of the final pattern as well as the flexibility of the technique. ${ }^{14-16}$

\footnotetext{
*Further author information: (Send correspondence to I.V. and G.G.) I.V.: E-mail:ilenia.viola@unile.it; G.G.: E-mail:giuseppe.gigli@unile.it; Fax number:+39 - $0832-298238$
} 
All in all, the growing success of bottom-up techniques is underlined by their potential application to a large class of liquid microchips and for opto-electronic or biological devices. ${ }^{17}$

As an example, the development of organic light-emitting diodes (OLEDs) technology ${ }^{5}$ has exploited, till now, also new techniques, as jet-printing and microfluidics, ${ }^{18}$ without yet a high direct control of the material deposition. ${ }^{6}$ Besides, in recent studies of Karthaus et al. the bottom-up techniques find application on OLEDs, made by random microdomes. ${ }^{19}$

In this paper, we present a controlled, well-defined pattern replication of a micrometer template driven by the surface free energy conditions and geometrical confinement. Besides, we show how the resolution of the final pattern is affected by the materials' chemical properties. Finally, we present an application of the technique to light-emitting devices, by realizing an OLED with an array of addressable pixels.

\section{SELF-ASSEMBLING APPROACH}

The proposed bottom-up approach, as a lithographic technique, relies on the differences in the liquid dynamics in confined conditions with respect to the behavior in bulk. Sub-millimeter systems, in fact, usually fulfill a fast diffusion regime, in which the dynamics is dominated by the surface interactions and localization phenomena. A significative length range is referred to the capillary dimension, ${ }^{12}$ strongly connected to the pressure drop in a capillary system*.

Self-organization patterning of organic materials usually refers to liquids in non-wetting case, that occurs when the spreading liquid retracts from the substrate forming a finite contact angle. ${ }^{20}$

The process can be quantified, from a thermodynamic point of view, in terms of surface free energies by introducing the spreading coefficient $S=\gamma_{s}-\gamma_{l}-\gamma_{l s}$, where $s, l$ and $l s$ are respectively referred to the surface tensions of the substrate, liquid and liquid-solid interface. In order to allow the occurrence of a spontaneous process, the free energy of the process must be negative. Therefore, in terms of surface energies $S>0$ is for a wettable surface, ${ }^{21,22}$ while $S<0$ indicates a dewetting process favoured by long-range forces (such as Van-der-Waals forces).

In the case of volatile liquids, dewetting is initiated by the solvent evaporation and the rupture process of homogeneous thin films, only weakly influenced by the geometrical constrains. The liquid rupture can be described by the interplay of two confinement induced-effects: a spontaneous destabilization of the liquid film (known as spinodal dewetting), driven by the attractive Van-der-Waals interactions, ${ }^{23}$ and a heterogeneous nucleation in the confining structure.

The dewetting process is then completed by droplet shrinking, finite boundaries of the geometric template and instabilities phenomena at the liquid interface. The former develops with a transport of materials outwards to compensate the evaporation. ${ }^{24}$ The latter ones characterize the final part of the process and are driven by polar (i.e. Hydrogen bond) and apolar forces (i.e. Van-der-Waals and dispersion forces) as well as by surface tension variations with temperature or liquid composition (Marangoni-type convective transport). ${ }^{25-27}$

*The upper limit for the capillary dimension is assumed as $l=\left(\frac{2 \gamma}{\rho g}\right)^{2}$, with $\gamma$ the liquid tension, $\rho$ the density and $g$ the gravitational acceleration. This expression is derived by the Laplace pressure drop in a capillary $\Delta p \sim \frac{2 \gamma}{R}$. 
Table 1: Solvent parameters

\begin{tabular}{|c|ccc|}
\hline \hline Solvent & Density $[\mathrm{g} / \mathrm{ml}]$ & Surface Tension $[\mathrm{mN} / \mathrm{m}]$ & Vapor pressure $[\mathrm{hPa}]$ \\
\hline $\mathrm{CHCl3}$ & 1.5 & 27.30 & 213 \\
$\mathrm{CH}_{2} \mathrm{Cl}_{2}$ & 1.3 & 27.50 & 465 \\
$\mathrm{C}_{3} \mathrm{H}_{6} \mathrm{O}$ & 0.8 & 25.20 & 233 \\
\hline \hline
\end{tabular}

\section{PATTERNING DEPOSITION}

The molecular patterned layer was realized by carrying on a controlled bottom-up experiment in non-wetting conditions.

We put $1 \mu \mathrm{l}$ of volatile solutions on a patterned grid fixed on a substrate. The $0.1 \% \mathrm{w} / v$ solutions were freshly prepared by the organic molecule N,N'-Bis(3-methylphenyl)-N,N'-diphenylbenzidine (TPD) (fig.1(a)) (Sigma-Aldrich) respectively in chloroform $\left(\mathrm{CHCl}_{3}\right)$, dichloromethane $\left(\mathrm{CH}_{2} \mathrm{Cl}_{2}\right)$ and acetone $\left(\mathrm{C}_{3} \mathrm{H}_{6} \mathrm{O}\right)$ (J.T. Baker). The substrates used are indium-tin-oxide (ITO) covered glasses (Vision Tek Systems LTD.), cleaned by ultrasonic waves in acetone, 2-propanol and deionized water (10 min for each step).

As template mesh, for the geometrical confinement, we used trasmission-electron-microscopy (TEM) calibration copper $(\mathrm{Cu})$ grid (400 mesh), that was set on glass/ITO substrate. Grids are characterized by a mesh of $40 \mu \mathrm{m}$ wide squared holes and $20 \mu \mathrm{m}$ wide bars. The grid was fasten by a suitable metallic gasket in order to fix it on the ITO-glass substrate and to avoid any possible distortions in the pattern. After a complete evaporation of the solvent, grid was removed by proper tweezers.

As we have described above, the confinement of the molecule could be probably attributed to the interplay between the characteristic parameters of the solution as well as of the two solid interfaces (ITO and $\mathrm{Cu}$ grid). ${ }^{13,28}$ It has been demonstrated that surface tension, substrate polarity, solvent evaporation rate or solvent dipole moment influence the beginning of a dewetting process as well as the morphology or the pattern roughness. ${ }^{28,29}$ Therefore, the flexibility of the technique, here described, consists in the possibility to modify size and morphology of the pattern in the final device, choosing selectively some characteristics of the materials used.

The lithographic procedure for fabricating a molecular micro-structured array on a glass/ITO substrate by molecular self-organization is schematized in figure 2 .

After the drop evaporation (fig.2(a)) the TPD layer can be realized under the bars (fig.2(b)) or inside the template holes (fig.2(c)), as function of the solvent properties (see table 1).

In the first case, the TPD is deposited from a $\mathrm{CHCl}_{3}$ solution. The molecular self-organized layer of TPD was characterized by optical microscopy (Vanox AHMT3, Olympus), in transmission mode, in order to verify the geometrical assembling and the pattern reproducibility (see fig.3).

In the second case, the topography (fig.4) of the molecular array was modified by changing the polarity and the vapor pressure of the solvent employed. Figure 4 reports a complementary pattern, in which organic molecules are confined inside the mesh squares, realized by depositing TPD from acetone $\left(\mathrm{C}_{3} \mathrm{H}_{6} \mathrm{O}\right)$ solution.

The flexibility of the self-organization approach we present, for the fabrication of molecular array, is well shown also by the pixel array obtained by depositing the TPD from dichloromethane $\left(\mathrm{CH}_{2} \mathrm{Cl}_{2}\right)$ 
solution (fig.5). With respect to the case of TPD deposited from chloroform (fig.3), the $\mathrm{CH}_{2} \mathrm{Cl}_{2}$ solution shows a pattern with a different resolution for the mesh bars, reproduced with a larger width.

In order to quantify, with sub-micrometer resolution, the morphology of the obtained structures we have performed atomic force microscopy (AFM, Solver Pro, NT-MDT)), in semi-contact mode. The pattern of TPD from $\mathrm{CHCl}_{3}$ solution, in particular, reproduces the original template with a U-shaped homogeneous profile of about $400 \mathrm{~nm}$ for the average height (fig.6). The U-shaped film profile results from the solvent evaporation and the mass transfer especially at the solid-liquid contact line. ${ }^{30}$ The AFM images highlight also the quality of deposition and the kind of spatial molecular aggregation in the layer. These features are strongly connected to the solvent peculiarity, as it is shown for the complementary pattern in acetone (fig.7).

\section{OLED FABRICATION}

The pixel arrays, obtained by the bottom-up lithographic approach (fig.8(a)), were used to realize OLED structures with an array of addressable active pixels.

To this aim we deposited by thermal evaporation $50 \mathrm{~nm}$ of 8-Hydroxyquinoline,aluminum salt $\left(\mathrm{AlQ} \mathrm{Q}_{3}\right.$ ) (fig.1(b)) (rate $=3 \div 5 \AA / \mathrm{s}$ ) on the patterned structure of TPD deposited from $\mathrm{CHCl}_{3}$ (fig.3). The $A l Q_{3}$ acts as the active layer on the TPD, acting as insulating layer due to the large thickness of $400 \mathrm{~nm}$. Finally, we deposited $50 \mathrm{~nm}$ of Calcium and $150 \mathrm{~nm}$ of Aluminum, acting as cathode (see fig.8(b)). The evaporation pressure was $P \simeq 10^{-6}$ mbar. The final OLED device was biased with a dc voltage of $10 \mathrm{~V}$ and the elctroluminescence was characterized by a CCD sensor working on a confocal laser scanning microscope (Olympus, FluoView1000). The optical images in figures 9(a) and 9(b) show the final patterned device before and after the dc bias. The green light emission, due to $A l Q_{3}$, is from the centre of each squared feature.

\section{CONCLUSIONS}

In conclusion we realized a controlled arrays of organic molecules by a surface tension driven technique, exploiting the combination both of the liquid instabilities during the evaporation, and the geometrical confinement, induced by a template mesh. The leading idea lies in exploiting the higher TPD structures under the bars as insulating barriers ${ }^{\dagger}$, thus allowing the fabrication of a disconnected array of addressable pixels.

The main advantages of the technique are the ease of realization and the flexibility on the resolution and the topography of the final pattern. This is promising for engineering nano and micro-patterns in thin film without the use of conventional lithographic processes, such as nanoimprinting and photolithography, which can damage the involved materials due to heating or UV exposure steps.

\section{Acknowledgments}

The authors gratefully acknowledge M. Cavallini and F. Biscarini (CNR-ISMN Bologna, Italy) for useful discussions.

\footnotetext{
${ }^{\dagger}$ The current density at low voltage, in OLED devices, in fact is usually proportional to $d^{-3}$. Therefore the height of the TPD patterned bars is such as to stop current flows.
} 


\section{REFERENCES}

1. K. Kargupta, A. Sharma, Langmuir, 18, pp. 1893-1903, 2002.

2. M. Cavallini, M. Murgia, F. Biscarini, Nano Lett., 1(4), pp. 193-195, 2001.

3. N. Tessler, G.J. Denton, R. H. Friend, Nature, 382, pp. 695, 1996.

4. M. Mazzeo, V. Vitale, F. Della Sala, M. Anni, G. Barbarella, L. Favaretto, G. Sotgiu, R. Cingolani, G.Gigli, Adv. Mater., 17(1), pp. 34, 2005.

5. C.W. Tang, S.A. Van Slyke, C.H. Chen, J. Appl. Phys., 65, pp. 3610-3616, 1989.

6. B.J. Matterson, J.M. Lupton, A.F. Safanov, M.G. Salt, W.L. Barnes, I.D.W. Samuel, Adv. Mat., 13(2), pp. 123-127, 2001.

7. T.R. Hebner, C.C Wu, D. Marcy, M.H. Lu, J.C. Sturm, Appl. Phys. Lett., textbf72, pp. 519,1998.

8. J.A. Calcaterra, K.B. Bennett Displays, 24(2), pp. 85,2003.

9. H. Aziz, Z. Popovic, S. Xie, A.M. Hor, N.X. Hu, C. Trip, G. Xu, Appl. Phys. Lett., 72, pp. 756, 1997.

10. D. Pisignano, S. D'Amone, G. Gigli, R. Cingolani J. Vac. Sc. Technol. B, 22(4), pp. 1759-1763, 2004.

11. X.M. Zhao, Y. Xia, G.M. Whitesides, J. Mater. Chem., 7, pp. 1069-1074, 1997.

12. S. Harkema, E. Schäffer, M.D. Morariu, U. Steiner, Langmuir, 19, pp. 9714-9718, 2003.

13. M. Massi, M. Cavallini, S. Stagni, A. Palazzi, F. Biscarini, Mater. Science and Engineer. C, 23, pp. 923-925, 2003.

14. M. Cavallini, F. Biscarini, M. Massi, A. Farran-Morales, D.A. Leigh, F. Zerbetto, Nano Lett., 2(6), pp. 635-639, 2002.

15. M. Brinkmann, S. Graff, F. Biscarini, Phys. Rev. B, 66, 165430, 2002.

16. O. Karthaus, N. Maruyama, X. Cieren, M. Shimomura, H. Hasegawa, T. Hashimoto, Langmuir, 16(15), pp. 6071-6076, 2000.

17. S. Herminghaus, A. Fery, S. Schlagowski, K. Jacobs, R. Seemann, H. Gau, W. Mönch, T. Pompe, J. Phys.: Condens. Matter, 12, pp. A57-A74, 2000.

18. M.L Chabinyc, W.S. Wong, K.E. Paul, R.A Street, Adv. Mater., 15(22), pp. 1903-1907, 2003.

19. O. Karthaus, C. Adachi, S. Kurimura, T. Oyamada, Appl. Phys. Lett., 84(23), pp. 4697, 2004.

20. T.G. Stange, D.F. Evans, W.A. Hendrickson, Langmuir, 13, pp. 4459-4465, 1997.

21. F. Brochard-Wyart, J. Daillant, Can. J. Phys., 68, pp. 1084-1088, 1990.

22. A. Faldi, R.J. Composto, K.I. Winey, Langmuir, 11, pp. 4855-4861, 1995.

23. A. Sharma, Langmuir, 9, pp. 861-869, 1993.

24. R.D. Deegan, O. Bakajin, T.F. Dupont, G. Huber, S.R. Nagel, T.A. Witten, Nature, 389, pp. 827-829, 1997.

25. M. Maillard, L. Motte, M.P. Pileni, Adv. Mat., 13(3), pp. 200-204, 2001.

26. O. Karthaus, L. Grasjö, N. Maruyama, M. Shimomura, CHAOS, 9(2), pp. 308-314, 1999.

27. O. Karthaus, T. Koito, M. Shimomura, Mater. Science and Engineering C, 8-9, pp. 523-526, 1999.

28. J. Zhao, S. Jiang, Q. Wang, X. Liu, X. Ji, B. Jiang, Appl. Surface Science, 236, pp. 131-140, 2004.

29. L.M. Pismen, Phys. Rev. E, 70, 021601, 2004.

30. X. Wang, M. Östblom, T. Johansson, O. Inganäs, Thin Solid Films, 449, pp. 125-132, 2004. 


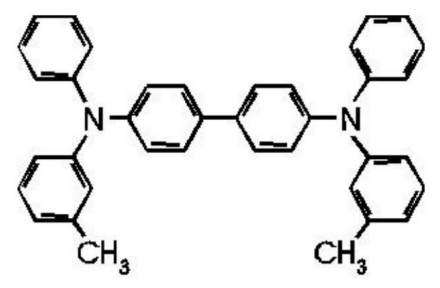

a)

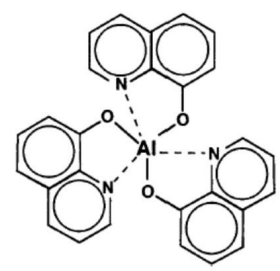

b)

Figure 1. Chemical structures of the organic molecules used in the experiments: a) N,N'-Bis(3-methylphenyl)N,N'-diphenylbenzidine (TPD); b) 8-Hydroxyquinoline,aluminum salt $\left(A l Q_{3}\right)$.

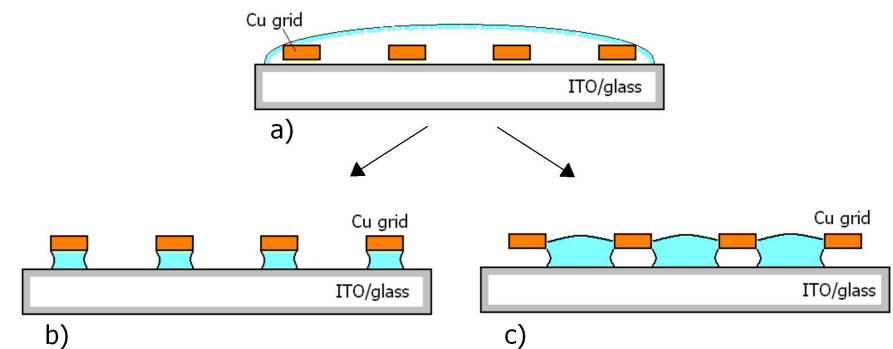

Figure 2. Sketch of the surface tension driven procedure, used for patterning a molecular layer: a) a drop of dilute solutions of volatile solvents $\left(\mathrm{CHCl}_{3}, \mathrm{CH}_{2} \mathrm{Cl}_{2}\right.$ and acetone) was put in the centre of a template mesh, set on a ITO/glass substrate; after the solvent evaporation the organic molecule (TPD) moves under the mesh bars to reproduce the original template on the substrate (b)) or is confined inside the template holes to form a negative pattern $(\mathbf{c}))$

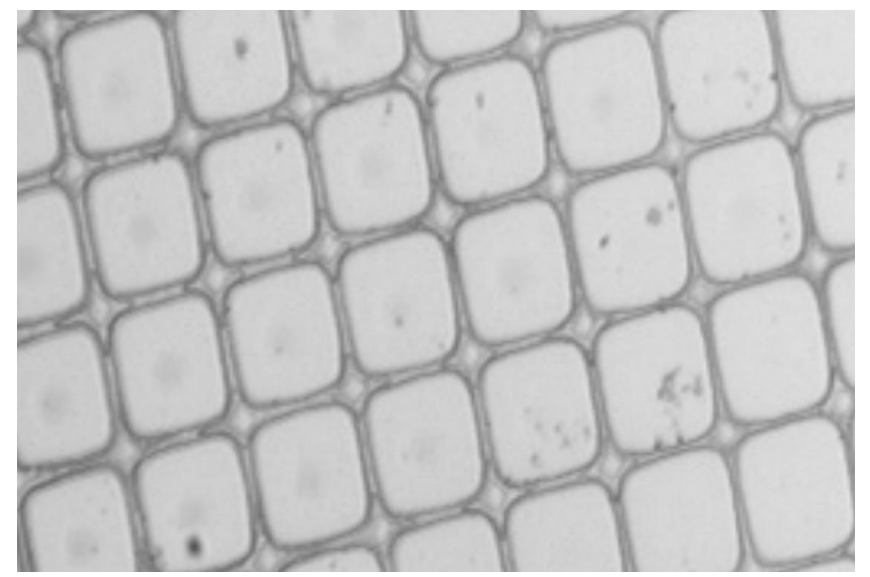

Figure 3. Optical image of the pattern realized by TPD in $\mathrm{CHCl}_{3}$. The pattern shows a template under the bar. 


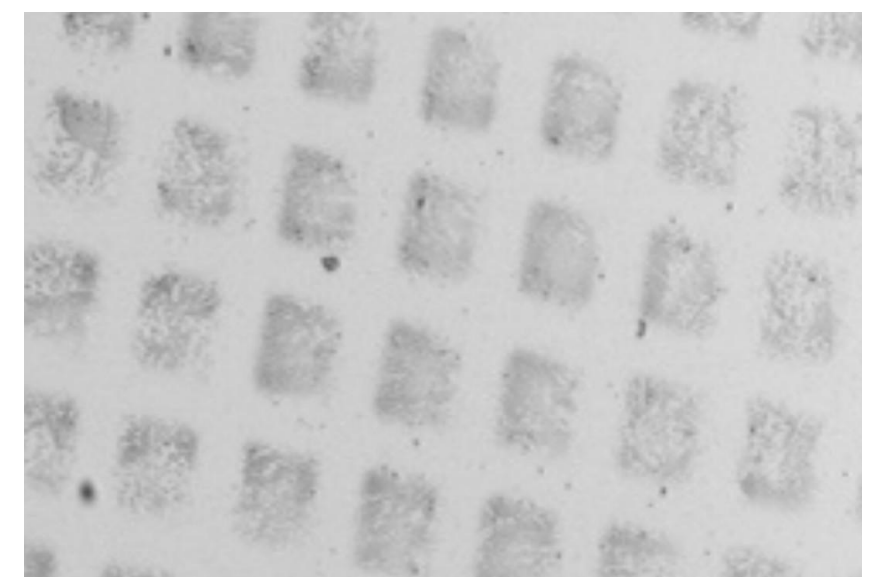

Figure 4. Optical image of the pattern realized by TPD in acetone. The polarity of acetone allows to obtain a complementary deposition with the molecule confined inside the holes.

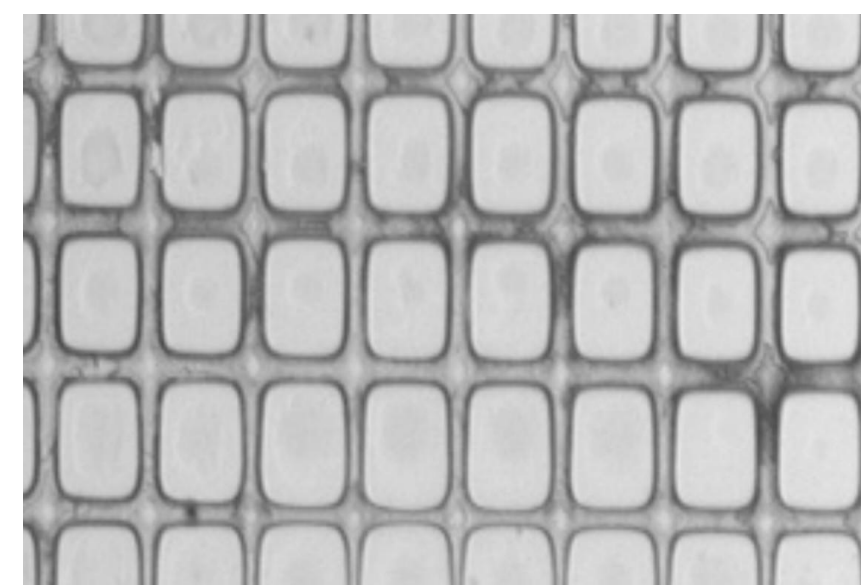

Figure 5. Optical image of the pattern realized by TPD in $\mathrm{CH}_{2} \mathrm{Cl}_{2}$. The pattern shows a template under the bar with a different resolution.
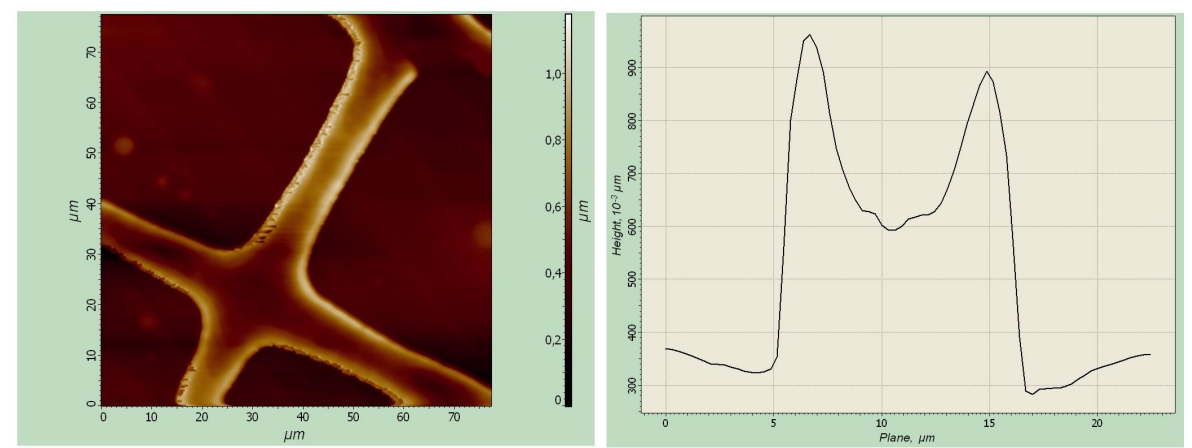

Figure 6. AFM image of the molecular array of TPD in $\mathrm{CHCl}_{3}$ obtained in nonwetting experimental conditions; the profile reveals a U-shape due to the material confinement towards the rims. 

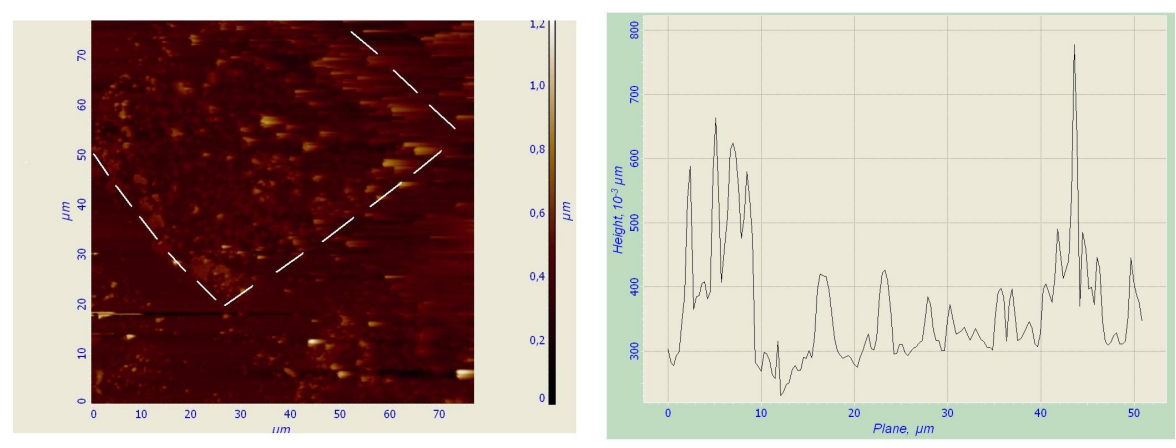

Figure 7. AFM image of the molecular array of TPD in acetone. The pattern reveals a complementary array in which organic molecules are confined inside the mesh squares.

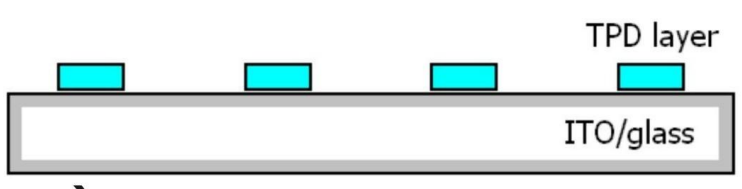

a)

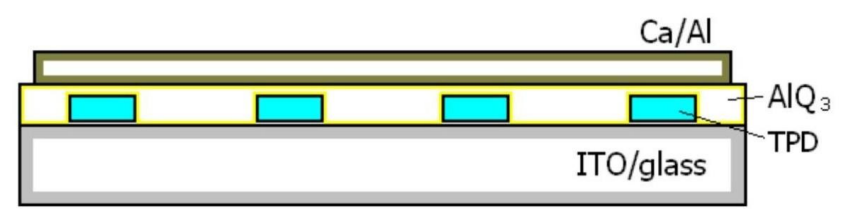

b)

Figure 8. c) The reproduced molecular layer on the substrate; d) the self-organized patterned substrate was used to fabricate an array of light-emitting devices (OLED). 


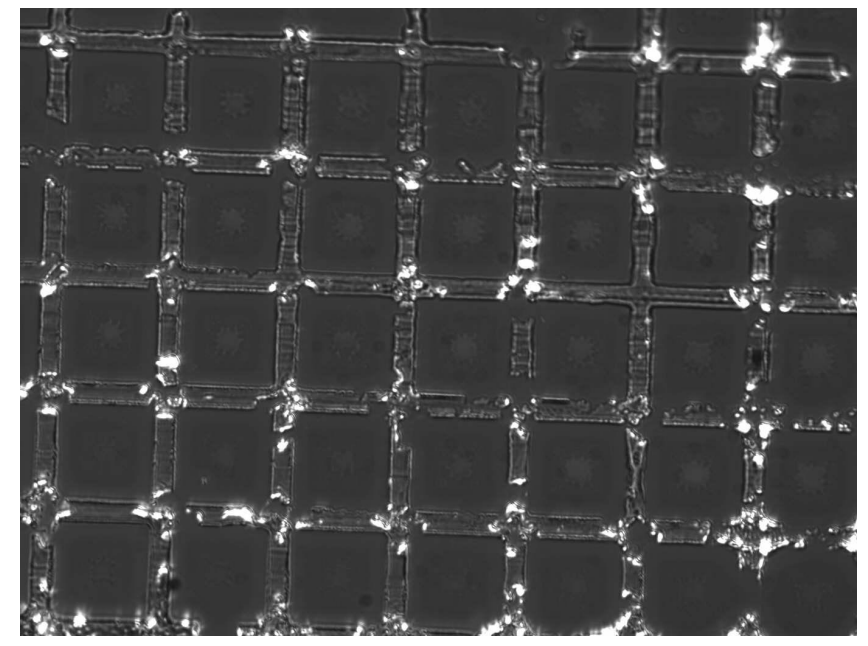

(a)

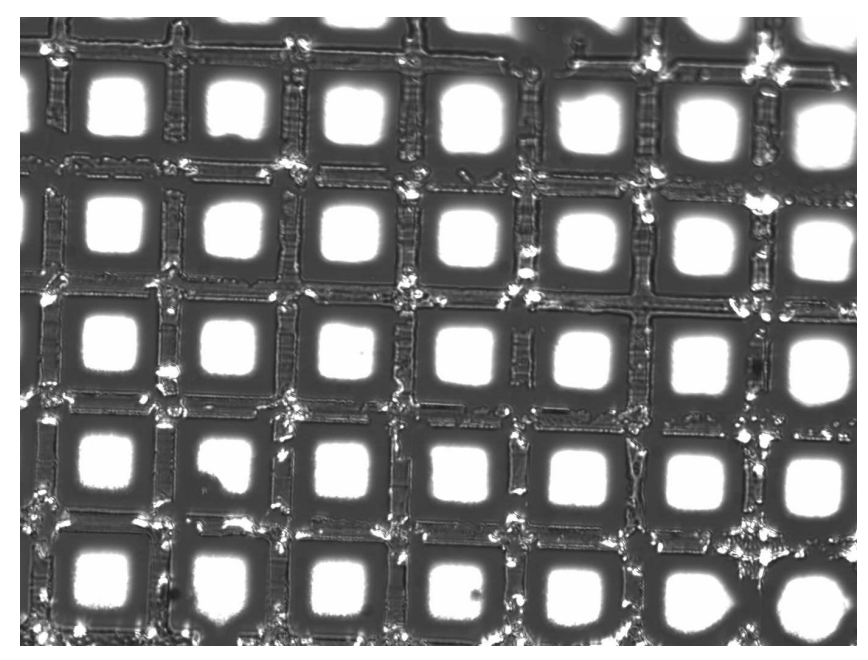

(b)

Figure 9. Optical images of the patterned OLED device, realized on a TPD array, before (a)) and after (b)) the light output driven with a dc voltage of $10 \mathrm{~V}$. 